\title{
Human Hand Movements in Skilled Motor Performance of a Virtual Curling Task
}

\author{
Yoshiyuki TANAKA $^{1)} \quad$ Kazuhiro MATSUSHITA $^{1)} \quad$ Toshio TSUJI $^{1)} \quad$ Nobuaki IMAMURA $^{2)}$ \\ 1) Graduate School of Engineering, Hiroshima University, JAPAN, \{ytanaka, tsuji\} @bsys.hiroshima-u.ac.jp \\ 2) Department of Engineering, Hiroshima Kokusai University, JAPAN
}

\begin{abstract}
The present paper develops a virtual curling system using robotic devices based on the sensory motor integration theory for the rehabilitation of disabled human sensorimotor system. In the training, a trainee manipulates the handle of an impedance-controlled robot and slides the stone toward the center of the house, as similar as an actual curing game. The developed system can estimate trainee's hand impedance during the curing task to quantitatively evaluate an adaptation ability of musculoskeletal characteristics of the upper extremity. To design a training program and an evaluation index of training effects, standard hand motion and its dynamic properties for the virtual curing task are clarified through a set of training experiments with 18 health volunteers.
\end{abstract}

\section{INTRODUCTION}

Recently, a number of advanced training systems using robotic devices with virtual reality have been developed [1][5]. Krebs et al. [1][2] developed the training system for extremities using an impedance-controlled robot to recover damaged sensory and/or motor functions, in which the trainee manipulates an end-effector to follow a target trajectory on the feedback display. Furusho et al. [4][5] developed the 3D rehabilitation system for upper limbs using ER actuators with highly safety and performance. It is expected that such advanced training systems would enable to evaluate training effects and to recover damaged motor function effectively. These previous studies, however, do not clarify reference properties of human movements for the programmed training task that will be required in designing a quantitative index to evaluate trainee's motion.

On the other hand, a human has highly developed sensory receptors and functions that allow him to survive and recognize various kinds of external information, and can regulate dynamic properties of his musculoskeletal system to achieve specific physical goals. Such dynamic properties of human movements can be expressed by mechanical impedance parameters: stiffness, viscosity, and inertia [6]-[8]. It suggests that the training in the regulation ability of human impedance would be effective for improving damaged motor functions of a trainee.

Tsuji et al. [9] proposed and validated an impedance training method focusing on human arm impedance regulation mechanism. The impedance training, however, can be applied only to static motions during maintenance of arm posture. In order to improve the impedance training method, they developed the virtual tennis training system using the impedance-controlled robot and the virtual reality for the purpose of skill-level training in dynamic motion [11][12]. The technique of virtual reality allows us to apply external disturbances and to change environmental characteristics in training. However, the trainee's end-point motion is limited in a straight trajectory and the level of difficulty on the task cannot be changed. In addition, they did not discuss the spatio-temporal trajectory of human hand during the training task depending on the learning effect and task condition.

In this paper, a virtual curling system using an impedancecontrolled robot with two D.O.F is developed, in which a trainee releases a stone toward a house displayed in the virtual space by operating the system. The primary subject is to clarify reference motor properties of the skilled player in the virtual curling task through a set of training experiments with healthy volunteers. This paper is organized as follows: Section II explains the developed virtual curling system and the measuring method of human hand impedance. Section III examines the changes of the spatio-temporal trajectory of human hand on the learning of the virtual curling task. Finally, the reference motion is discussed from the additional experimental results with the skilled subjects in Section IV.

\section{Virtual CuRling System}

\section{A. System Structure}

Fig. 1 shows an overview of the virtual curing system. The system is composed of an impedance-controlled robot for providing virtual force to a trainee, a DSP instrument for robot control and signal processing, and a biofeedback display for presenting virtual curing game.

The robot is composed of two linear motor tables with one degree of freedom (Nihon Seikou Coop., maximum force: $x$ axis, $100[\mathrm{~N}] ; y$ axis, $400[\mathrm{~N}])$ that are placed orthogonally in order to carry out the two-dimensional hand motion exercise. During training, hand force generated by the trainee is measured by a six-axis force/torque sensor (Nitta Co. Ltd., resolution: force $x$ and $y$ axes, $0.005[\mathrm{~N}] ; z$ axis, $0.15[\mathrm{~N}]$; torque, $0.003[\mathrm{Nm}]$ ) attached on the handle of robot. The handle position is measured by an encoder built in the linear motor tables (resolution: $x$ and $y$ axes, $1.0[\mu \mathrm{m}]$ ).

The DSP instrument (A\&D Co. Ltd., AD5410) is utilized for realizing the precise impedance-control of robot motion in real-time and for estimating human impedance properties during a virtual curling task. 


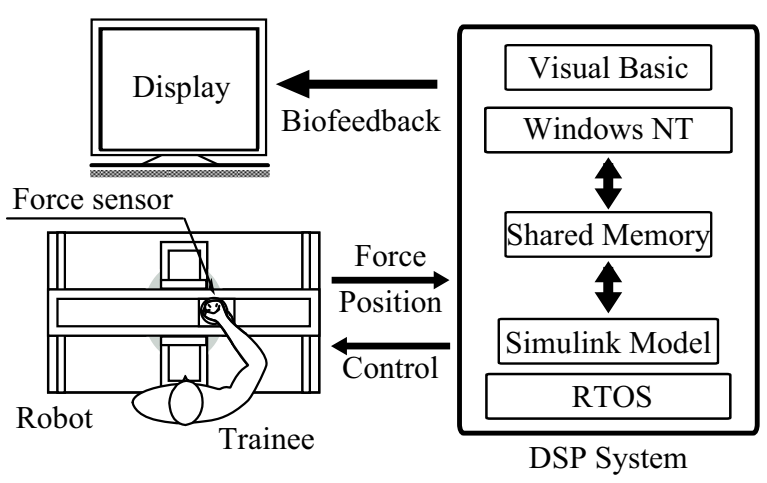

Fig. 1. An overview of the impedance training system

The feedback display presents the task space of virtual curling as shown in Fig. 2. The stone in the screen moves corresponding to the handle position, and the scene changes when the stone is in the neighborhood of the house.

\section{B. Virtual Curling Model}

Fig. 3 illustrates a model of the virtual curling task. A trainee is asked to move a stone with radius $R_{s}$ to the center of a house with radius $R_{h}$ by manipulating the robot handle, while the stone is automatically separated from the handle when it passes the release line with $y=d$. The initial position of the stone is in front of the center of a house $O_{h} \in \Re^{2}$, so the trainee needs to generate a straight hand trajectory in the two-dimensional task space.

In the virtual curing, dynamics of human hand movements can be given by

$$
F_{e}= \begin{cases}\left(M_{r}+M_{s}\right) \ddot{X}_{e}+\left(B_{r}+B_{\text {floor }}\right) \dot{X}_{e} & (0 \leq y \leq d) \\ M_{r} \ddot{X}_{e}+B_{r} \dot{X}_{e} & (y>d)\end{cases}
$$

where $F_{e} \in \Re^{2}$ is the hand force generated by a trainee, $X_{e} \in$ $\Re^{2}$ the hand position of a trainee, $M_{r}=\operatorname{diag} .\left(m_{r}, m_{r}\right) \in$ $\Re^{2 \times 2}$ and $B_{r}=\operatorname{diag} .\left(b_{r}, b_{r}\right) \in \Re^{2 \times 2}$ are the inertia and viscosity of the handle, $M_{s}=\operatorname{diag} .\left(m_{s}, m_{s}\right) \in \Re^{2 \times 2}$ is the inertia of the stone, and $B_{\text {floor }}=\operatorname{diag} .\left(b_{\text {floor }}, b_{\text {floor }}\right) \in \Re^{2 \times 2}$ is the viscosity between the stone and the floor.

Dynamic behavior of the stone $X_{s} \in \Re^{2}$ after passing the release line $(y>d)$ is calculated as:

$$
M_{s} \ddot{X}_{s}+B_{\text {floor }} \dot{X}_{s}=0
$$

Therefore, the task performance is strongly influenced by the stone velocity at $y=d$. As contacting the wall parallel to the $x / y$ axis, the stone motion is produced by reversing the stone velocity to the $y / x$ axis.

\section{Human Impedance Measurement}

Humans perform a target task successfully by regulating hand impedance properties to desired ones in preparation for the task before motions based on prior experience [11] [12]. Therefore, human impedance measured just before and after motions may reflect the intention of human movements depending on the target task.

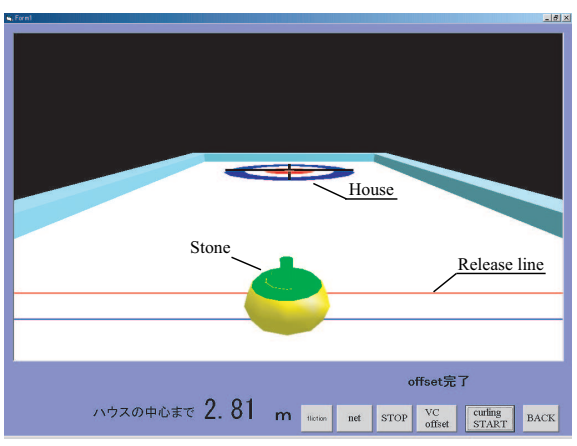

(a)

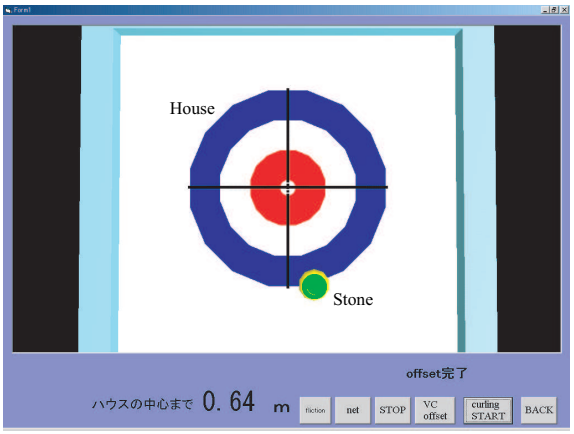

(b)

Fig. 2. Example of a biofeedback display in Virtual Curling

Let us consider multi-joint movements by the human upper extremity in the l-dimensional task space. When the subject's end-point is displaced from its equilibrium by a small disturbance with a short duration as shown in Fig. 4, dynamic characteristics of the hand can be expressed with an impedance model [8] as

$$
M_{e} \ddot{X}_{e}(t)+B_{e} \dot{X}_{e}(t)+K_{e}\left(X_{e}(t)-X_{v}(t)\right)=-F_{e}(t),
$$

where $F_{e}(t) \in \Re^{l}$ denotes the hand force applied to the environment; $X_{e}(t) \in \Re^{l}$ the hand position; $X_{v}(t) \in \Re^{l}$ the virtual trajectory; and $M_{e}, B_{e}$, and $K_{e} \in \Re^{l \times l}$ represent hand inertia, viscosity and stiffness, respectively. Assuming that $X_{v}(t)$ is constant, the following equation of hand dynamics can be derived from (3):

$$
M_{e} d \ddot{X}(t)+B_{e} d \dot{X}(t)+K_{e} d X(t)=-d F_{e}(t),
$$

where $d X(t)=X_{e}(t)-X_{e}\left(t_{0}\right), d F(t)=F_{e}(t)-F_{e}\left(t_{0}\right)$, and $t_{0}$ denotes the time when the disturbance is applied to the hand. In this model, the hand impedance matrices can be estimated from the measured hand position $X_{e}(t)$ and the hand force $F_{e}(t)$, induced by the external disturbance, with the least squares method.

Mechanical impedance properties of a known spring-mass system were measured to validate performance of the developed system, where the specified values of spring stiffness were $85,177,300,442,560[\mathrm{~N} / \mathrm{m}]$ and inertia of masses were $0.66,1.15,1.66,2.3$ [kg]. Fig. 5 shows an example of the measured signals for estimating mechanical impedance 


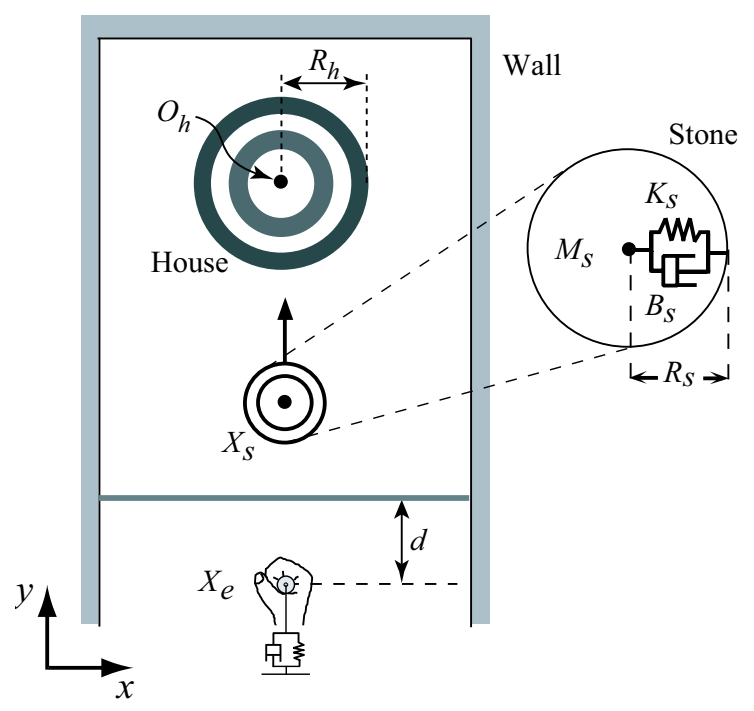

Fig. 3. Model of the virtual curling task

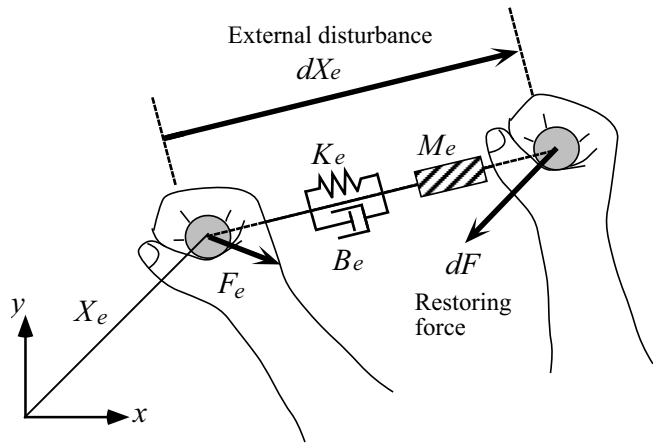

Fig. 4. Schematic description of the hand impedance

properties. The panels (a), (b), and (c) express time histories of the displacement of an end-point $d y(t)$, velocity $d \dot{y}(t)$, and acceleration $d \ddot{y}(t)$ caused by external disturbance in the order from the top. The solid line in the bottom panel (d) represents measured force together with estimated force (broken line) calculated from Eq.(4) with measured signals and estimated impedance parameters. It can be said that the experimental system accurately estimates mechanical impedance properties since the solid line almost coincides with the broken one. The standard deviations of estimated errors for stiffness and inertia were less than $4.93[\mathrm{~N} / \mathrm{m}]$ and $0.007[\mathrm{~kg}]$ in the $x$ axis, 4.66 $[\mathrm{N} / \mathrm{m}]$ and $0.027[\mathrm{~kg}]$ in the $x$ axis, respectively, within the specified conditions.

\section{Human Hand Movements In SKILl-ACQUiSition}

Experiments were carried out with 18 healthy volunteers in this study. The centers of stone and house were put on the $y$ axis, and the distance between them was set at $6[\mathrm{~m}]$.

Fifty trials were set for each normal subject to investigate the changes of human hand movements in the process of skillacquisition after brief explanations on the virtual curling task
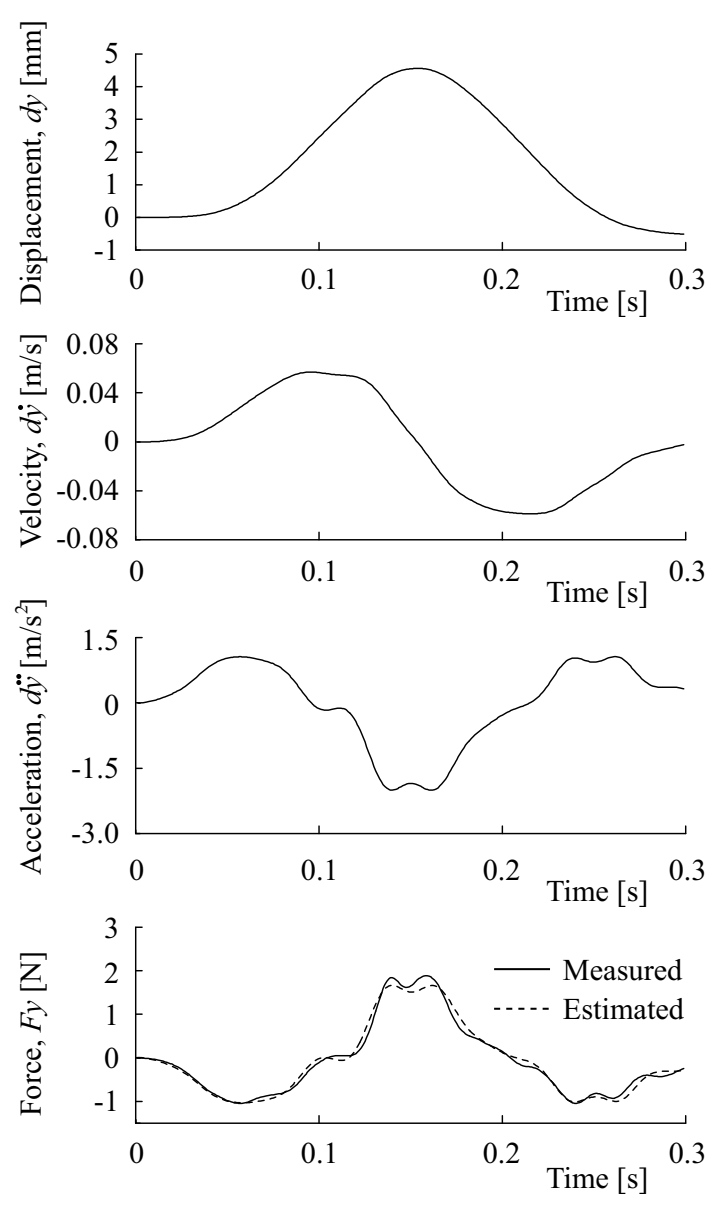

Fig. 5. An example of measured signals in the impedance estimation

and the operating system. A subject stood in front of the system so that his shoulder joint, his hand, and the center of a house were arranged in a straight line, and he was instructed to release and stop the stone at the center of the house by manipulating the robot handle as he hoped.

The center and radius of the house were set at $O_{h}=$ $(0.0,6.0)^{\mathrm{T}}, R_{h}=0.6[\mathrm{~m}]$; the inertia and viscosity of the handle at $m_{r}=5[\mathrm{~kg}], b_{r}=1.0[\mathrm{Ns} / \mathrm{m}]$; the impedance of the stone at $m_{s}=15[\mathrm{~kg}], b_{s}=100[\mathrm{Ns} / \mathrm{m}], k_{s}=10000[\mathrm{~N} / \mathrm{m}]$; the viscous friction between the stone and the floor at $b_{\text {floor }}=1.0$ $[\mathrm{Ns} / \mathrm{m}]$. Under this parameter setting, the ideal stone velocity to the $y$ axis at $y=d$ is calculated from Eq. (2) at $y_{e}^{*}=4.01$ $[\mathrm{m} / \mathrm{s}]$. The task performance for the subjects was evaluated by the two quantitative indices: an absolute positional error between the released stone and the center of the house; $E_{p}$, and an absolute velocity error between the releasing stone and $y_{e}^{*}, E_{v}$.

Fig. 6 shows trial histories of the task performance, $E_{p}$ and $E_{v}$, and the angle between motion direction of the stone and the $y$ axis just as releasing the stone, $\theta$ for Subject A. It suggests that the subject became to move the handle straightly toward the center of the house with the ideal hand velocity as increasing the trial number so that the stone could stop near 


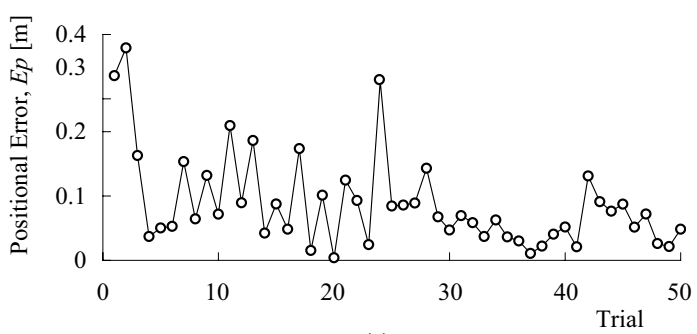

(a)

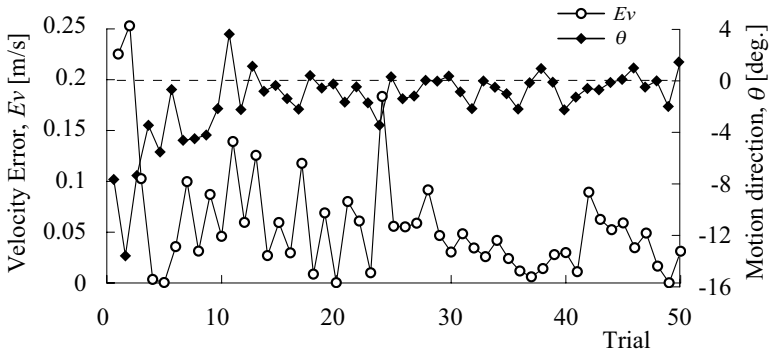

(b)

Fig. 6. Changes of the task performance with respect to the trial number

the center of the house.

Fig. 7 shows hand movements of Sub. A measured in the initial five trials (Trial No. 1, 2, . 5, 5), the middle five trials (Trial No. 23, 24, $\cdots, 27$ ), and the last five trials (Trial No. 46, $47, \cdots, 50$ ). The figure (a) shows the hand trajectories where the origin is set at the start point of hand movements, the figure (b) shows the hand velocity profiles along the $y$ axis where the initial time denotes the time when the stone was separated from the handle. The subject gradually decreases the errors with respect to the trial number of the release motion while generates almost the same velocity profile in virtual curing. Note that the hand velocity just at releasing the stone almost converges to the ideal value $y_{e}^{*}$ in the last five trials.

Fig. 8 presents the evaluation results and the correlation of hand velocity profiles with the averaged one for all subjects in each stage of the learning, in which a mean value with a standard deviation of the five trials are plotted. Although there exist some individual differences, all subjects acquire the skill in the virtual curing though the almost same learning process.

\section{AdAPTATION ABILITy FOR ENVIRONMENTAL PROPERTIES}

The further experiment was performed for the three skilled subjects (Subs. A, B, C) by changing the inertia of the handle as $m_{r}=5,10,15[\mathrm{~kg}]$ to extract a reference motion in the virtual curling task. The number of trials for each value of handle inertia was set at 20, and the other experimental parameters were same as the preliminary tests described in the previous section.

Fig. 9 shows the hand velocity and force profiles toward the $y$ axis measured during the last trial according to the different values of handle inertia $m_{r}$. Note that the subjects did not change their hand velocity profile with respect to $m_{r}$ although dynamic properties of a human-robot system were different

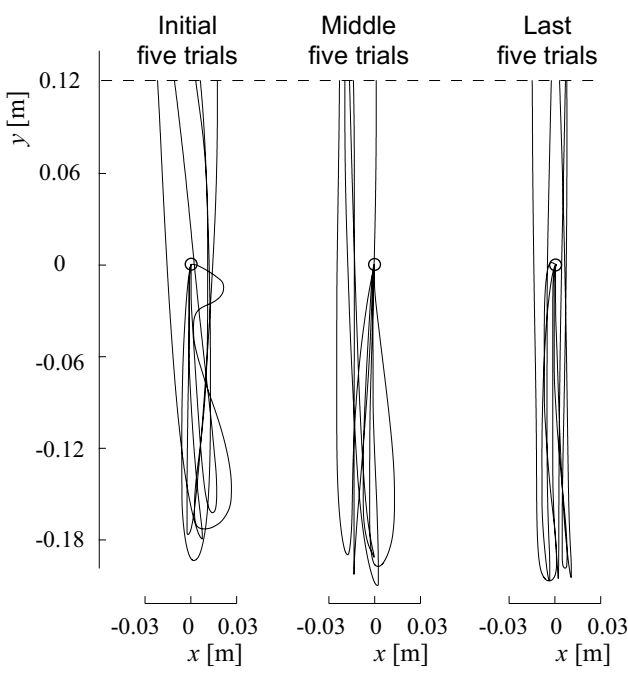

(a)
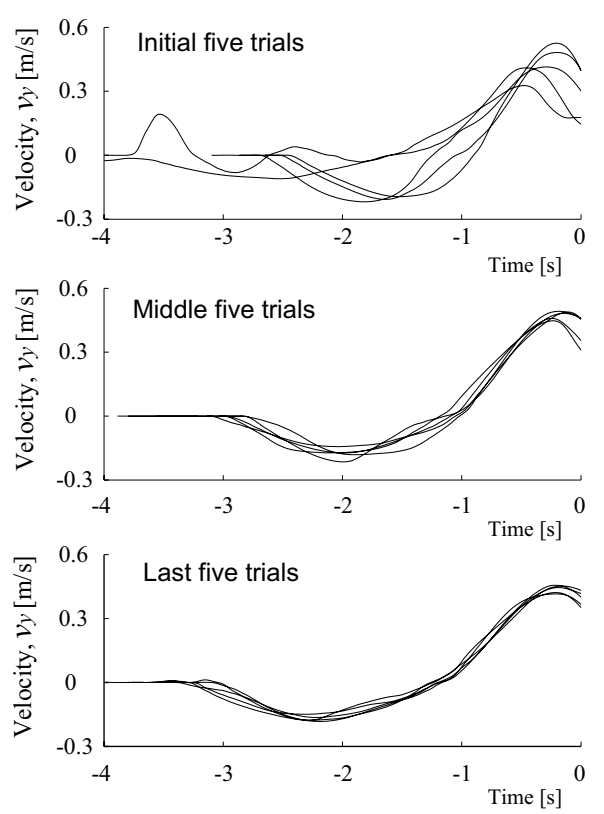

(b)

Fig. 7. Spatio-temporal characteristics of hand movements by Subject A during virtual curing task

during release motion. These results strongly suggest that a skilled player can simulate dynamic behavior of the stone after separating from the handle and can control his musculoskeletal system to generate the suitable hand velocity profile with the ideal velocity $y_{e}^{*}$ at the release line $y=d$. Accordingly, it can be said that the hand velocity profile should be utilized as a training target in virtual curing.

To quantitatively evaluate such skillful hand movements in the virtual curling, human hand impedance was measured just before motion, at the turn from back to push motions, and just after motion according to the different three values of the handle inertia.

Fig. 10 shows the mean and standard deviation of measured 


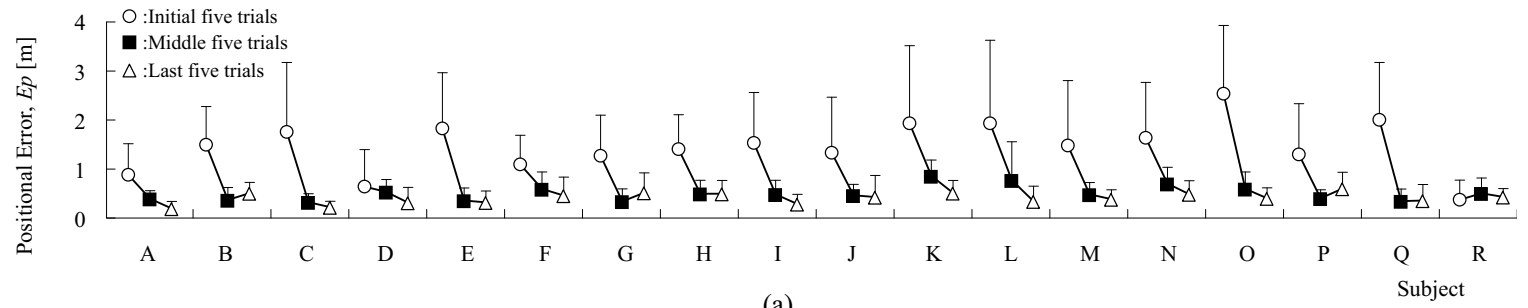

(a)

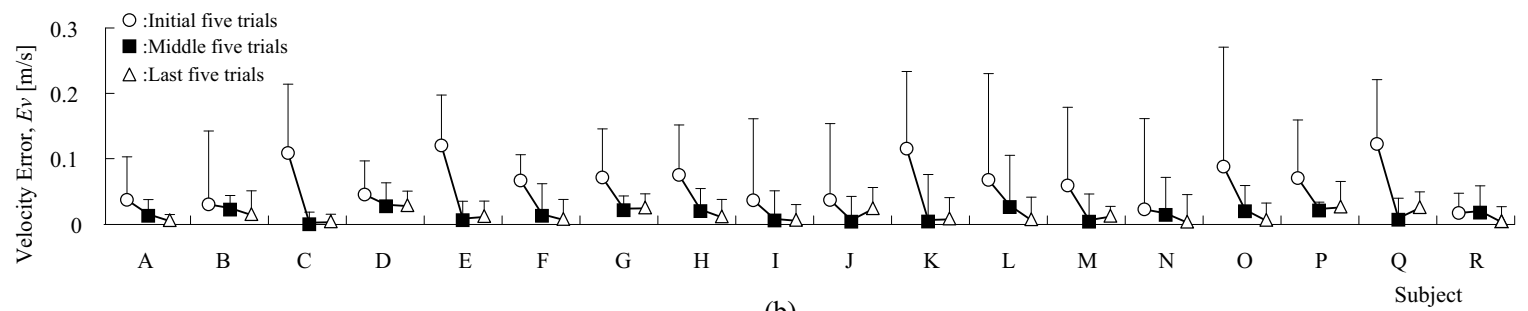

(b)

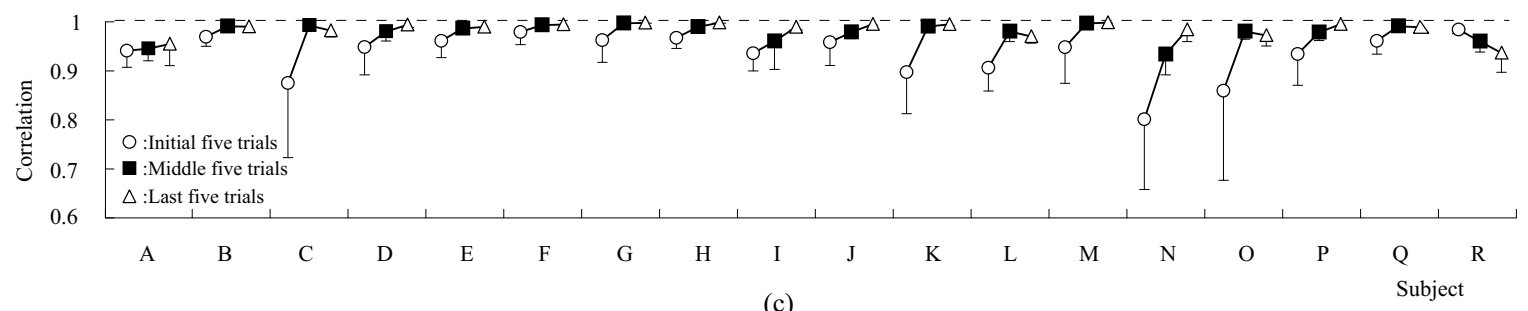

(c)

Fig. 8. Skill acquisition process of a virtual curling task for all subjects
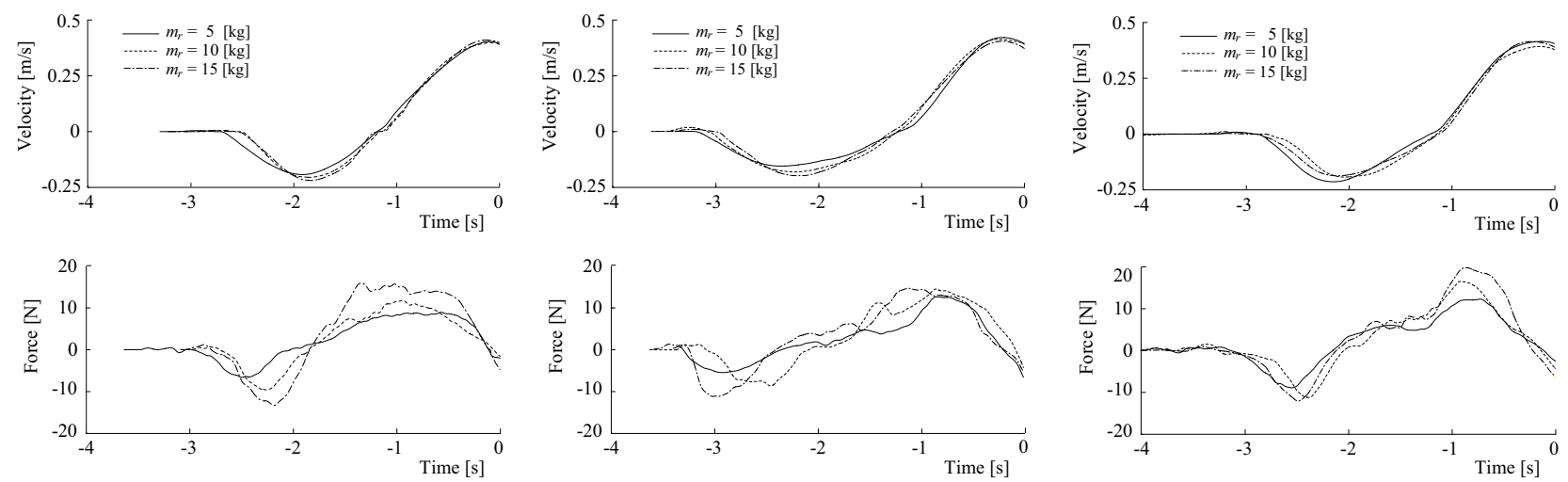

(b) Sub. B

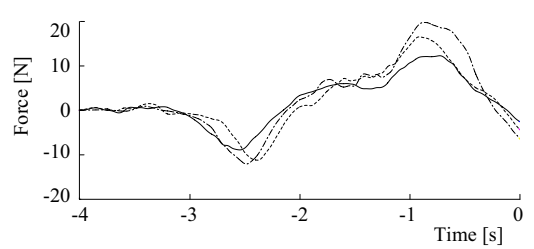

(c) Sub. C

Fig. 9. Hand velocity profiles for the different inertia values of robot handle

hand impedance for the five trials by Sub. A. The subject actively changes his hand impedance to the $y$ direction during tasks. He decreases hand viscoelastic properties at the turn to smoothly reverse the hand motion direction from back to forward, while increases after motion to make a starched arm posture stable. The same tendencies can be observed in the all subjects.

Table I shows the mean values of the natural frequency calculated from the measured hand impedance for each motion direction. The subjects increase the natural frequency to the $x$ direction, $\omega_{x}$, at the turn while decrease to the $y$ direction, $\omega_{y}$. As increasing the handle inertia, $\omega_{x}$ tends to become small while $\omega_{y}$ does large.

A series of the experimental results demonstrate that a trainee can train his fundamental ability required for the motor control in the virtual curing task: i) Perception ability of task environments, ii) Motion planning ability of a suitable hand trajectory based on stone dynamics, and iii) Regulation ability of his hand motion properties. Future research will be directed to design a quantitative evaluation index on the perception ability, motor planning ability, and motor control properties on the base of the experimental findings obtained. 


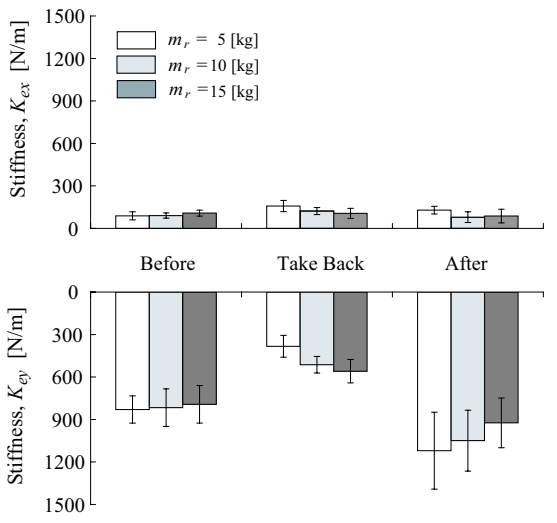

(a)

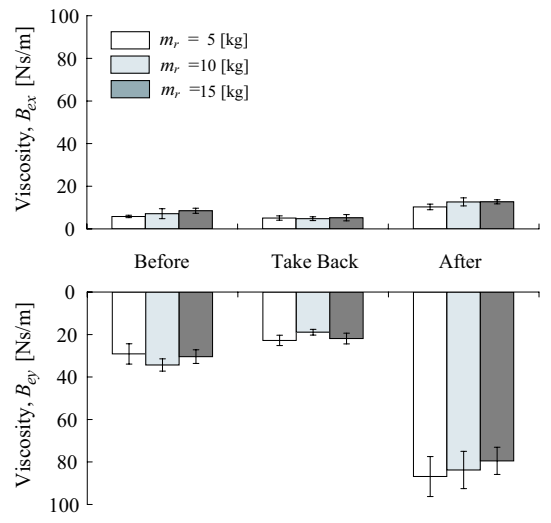

(b)

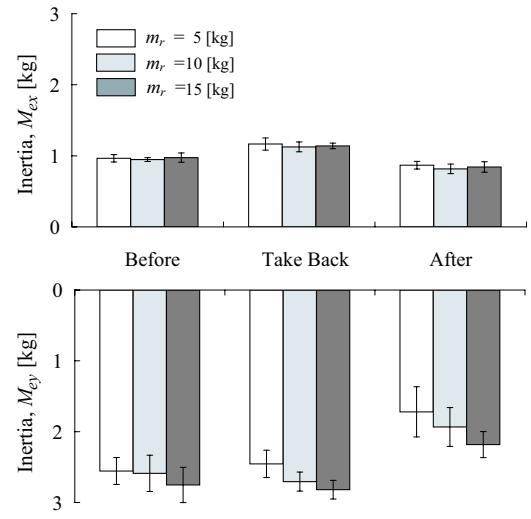

(c)

Fig. 10. Hand impedance parameters measured during tasks depending on the handle inertia

TABLE I

NATURAL ANGULAR FREQUENCY OF HAND MOTION B Y THE SUBJECTS

\begin{tabular}{cc|rc|rc|rc}
\hline & & \multicolumn{2}{|c|}{ Before } & \multicolumn{2}{|c|}{ Take Back } & \multicolumn{2}{c}{ After } \\
& $m_{r}[\mathrm{~kg}]$ & \multicolumn{1}{c|}{$\omega_{x}$} & $\omega_{y}$ & \multicolumn{1}{c|}{$\omega_{x}$} & $\omega_{y}$ & \multicolumn{1}{c}{$\omega_{x}$} & $\omega_{y}$ \\
\hline \hline & 5 & 9.58 & 18.02 & 11.64 & 12.49 & 12.19 & 25.52 \\
Sub. A & 10 & 9.78 & 17.75 & 10.43 & 13.77 & 9.87 & 23.29 \\
& 15 & 10.53 & 16.97 & 9.65 & 14.08 & 10.17 & 20.57 \\
\hline & 5 & 14.56 & 14.82 & 14.63 & 11.55 & 10.80 & 17.75 \\
Sub. B & 10 & 10.76 & 13.57 & 12.97 & 13.96 & 10.15 & 20.37 \\
& 15 & 9.53 & 14.69 & 11.68 & 14.28 & 10.14 & 22.12 \\
\hline & 5 & 9.81 & 13.29 & 13.33 & 10.53 & 8.82 & 17.12 \\
Sub. C & 10 & 12.19 & 13.84 & 12.23 & 12.27 & 9.84 & 23.01 \\
& 15 & 12.86 & 15.92 & 12.14 & 13.22 & 9.90 & 24.76 \\
\hline
\end{tabular}

\section{Conclusions}

This paper developed the virtual curling system using the impedance-controlled robot as a first step of realizing a novel upper arm rehabilitation system based on human sensorimotor properties. A series of the preliminary experiments with the 18 healthy volunteers demonstrates the following distinctive characteristics in the virtual curling as:

- A skilled player finds the ideal release velocity for the specified stone dynamics.

- A skilled player generates a unique hand velocity profile with a straight trajectory for the specified stone dynamics even when dynamic properties of the overall system were changed.

- A skilled player increases his hand impedance to the motion direction.

In the future research, the verification of the proposed training methodology will be carried out with the corporation of patients in motor disorders.

\section{ACKNOWLEDGMENT}

This research work was supported in part by a Grant-inAid for Scientific Research from the Japanese Ministry of Education, Science and Culture (15360226 and 18760193), and the 21st Century COE Program of JSPS (Japan Society for the Promotion of Science) on Hyper Human Technology toward the 21st Century Industrial Revolution. The authors would like to appreciate the kind supports of the A\&D Co. Ltd. on the DSP instrument.

\section{REFERENCES}

[1] H. I. Krebs, N. Hogan, M. L. Aisen and B. T. Volpe: "Robot-aided neurorehabilitation," IEEE Transactions on Rehabilitaion Engineering, Vol. 6, No. 1, pp. 75-87, 1998.

[2] H. I. Krebs, B. T. Volpe, M. L. Aisen and N. Hogan: "Increasing productivity and quality of care: Robot-aided neuro rehabilitation," Jurnal of Rehabilitation Reserch and Development, Vol.37, No.6, pp. 639-652, 2000.

[3] Y. Okajima, N. Tanaka, M. Hasegawa, N. Uchida, A. Kimura, Y. Tomita, T. Horiuchi, M. Kondo, T. Sakaki: "Therapeutic Exercise Machine: Soft Motion by the Impedance Control Mechanism," Sogo Rehabilitation, Vol. 26, No. 4, pp. 363-369, Igaku-shoin Ltd., 1998. (In Japanese)

[4] M. Sakaguchi, J. Furusho, and E. Genda: "Basic Study on Development of Rehabilitation Training System Using ER Actuators," Journal of the Robotics Society of Japan, Vol. 19, No.5, pp. 12-619, 2001.

[5] J. Furusho, K. Koyanagi, K. Nakanishi, U. Ryu, S. Takenaka, A. Inoue, K. Domen, and K. Miyakoshi: "Development of A 3-D Rehabilitation System for Upper Limbs Using ER Actuators in a NEDO Project," International Journal of Modern Physics B, Vol. 19, No. 7-9, pp.15911597, 2005.

[6] F. A. Mussa-Ivaldi, N. Hogan and E. Bizzi: "Neural, mechanical and geometric factors subserving arm in humans," Journal of Neuroscience, Vol. 5, No. 10, pp. 2732-2743, 1985.

[7] J. M. Dolan, M. B. Friendman, and M. L. Nagarka: "Dynamics and loaded impedance of numan arm posture," IEEE Transaction on Systems, Man, and Cybernetics, Vol. 23, No. 3, pp. 698-709, 1993.

[8] T.Tsuji, P.G.Morasso, K.Goto, K.Ito: "Human hand impedance characteristics during maintained posture," Biological Cybernetics, Vol. 72, pp. 457-485, 1994.

[9] T. Tsuji, Y. Kanji, T. Kato, M. Kaneko, and S. Kawamura: "Impedance Training: Can We Regulate Our Hand Impedance Through Training?," Transactions of the Society of Instrument and Control Engineers, Vol.35, No.10, pp. 1300-1306, 1999. (in Japanese)

[10] T.Tsuji, Y. Tanaka, T. Abe, and H. Miyaguchi: "Human Impedance Perception through Sensory-Motor Integration," Journal of Robotic and Mechatronics, Vol.15, No.2, 192/199, 2003.

[11] T.Tsuji, Y.Sumida, M.kaneko, S.kawamura: "A Virtual Sports System for Skill Training," Journal of Robotics and Mechatronics, Vol. 13, No. 2, pp. 168-175, 2001.

[12] T. Tsuji, Y. Takeda and Y. Tanaka: "Analysis of Mechanical Impedance in Human Arm Movements using a Virtual Tennis System," Biological Cybernetics, Vol. 91, No. 5, pp. 295-305, 2004.

[13] N. Hogan: "Impedance Control: An approach to Manipulation, Parts I, II, III," ASME Journal of Dynamic Systems, Measurement, and Control, Vol. 107, No. 1, pp. 1-24, 1985. 